\title{
Editorial: Special Issue on Algorithms and Computation
}

\author{
Seok-Hee Hong ${ }^{1}$
}

Published online: 12 April 2018

(C) Springer Science+Business Media, LLC, part of Springer Nature 2018

This special issue contains a selection of four papers from the 27th Annual International Symposium on Algorithms and Computation (ISAAC 2016), which was held on December 12-14, 2016 in Sydney, Australia. The ISAAC 2016 program committee accepted 62 papers among 155 high-quality submissions from 36 countries, after a rigorous review process. Among those accepted papers, the following four papers in the area of Algorithms and Computation were selected and invited to this special issue, based on their evaluation by the program committee. The articles have undergone rigorous peer-review according to the journals high standards.

This issue consists of four papers that represent recent advances in various areas of Algorithms and Theory of Computation research. The first paper, "Optimal Composition Ordering Problems for Piecewise Linear Functions” by Y. Kawase, K. Makino and K. Seimi, introduced optimal composition ordering problems with two settings (total and partial compositions) and input functions (monotone and almost linear), and studied their computational complexity. They presented polynomial time algorithms for the maximum total (partial) composition ordering problems when the input functions are monotone linear, and for the maximum total composition ordering problem when the input functions have specific properties. They also proved that there exists no constant-factor approximation algorithm for the maximum total (partial) composition ordering problems, even if input functions are monotone piecewise linear functions with at most two pieces.

The second paper, "Degree-Constrained Orientation of Maximum Satisfaction: Graph Classes and Parameterized Complexity" by H. Bodlaender, H. Ono and Y. Otachi, studied the MAX $W$-LIGHT (MAX $W$-HEAVY) problems for an undirected

Seok-Hee Hong

seokhee.hong@sydney.edu.au

1 School of Information Technologies, University of Sydney, Sydney, NSW 2006, Australia 
graph to assign a direction to each edge so that the number of vertices of outdegree at most (resp. at least) $W$ is maximized, known as NP-hard even for fixed $W$. They showed that for any fixed constant $W$, MAX $W$-HEAVY can be solved in linear time for hereditary graph classes for which treewidth is bounded by a function of degeneracy, including chordal graphs, circular-arc graphs, $d$-trapezoid graphs, chordal bipartite graphs, and graphs of bounded cliquewidth. They presented a dynamic programming approach to show that the MAX $W$-LIGHT problems can be polynomial-time solvable for graphs of bounded clique-width. They also studied the parameterized complexity of the problems and showed some tractability and intractability results.

The third paper, "On $(1, \epsilon)$-Restricted Max-Min Fair Allocation Problem" by T-H. Chan, Z.G. Tang and X. Wu, investigated the Max-Min fair allocation problem in which a set of $m$ indivisible items are to be distributed among $n$ agents such that the minimum utility among all agents is maximized. They considered the $(1, \epsilon)$-restricted Max-Min fair allocation problem in which each item $j$ is either heavy or light, and showed that the $(1, \epsilon)$-restricted case is also NP-hard to approximate within any ratio smaller than 2. Using the configuration-LP, they estimated the optimal value of the problem within a factor of $3+\delta$, for any $\delta>0$, and then obtained a quasi-polynomial time $(3+4 \epsilon)$-approximation algorithm and a polynomial time 9-approximation algorithm.

The final paper, "The Subset Assignment Problem for Data Placement in Caches" by S. Ghandeharizadeh, S. Irani and J. Lam, introduced the Subset Assignment problem to model the problem of managing a cache composed of banks of memory with varying cost/performance, where the number of data objects (items) $n$ is very large and the number of memory banks (bins) $d$ is a small constant, to determine an optimal assignment in time that minimizes dependence on $n$. They focussed on an efficient solution to the LP relaxation as the number of fractionally assigned items will be at most $d$, and presented an algorithm to solve the LP relaxation in time $O\left(3^{d} d+1(d) n \log (n) \log (n C) \log (Z)\right)$, where $Z$ is the maximum item size and $C$ is the maximum storage cost.

We wish to thank the authors for submitting their papers to the special issue. We also thank the referees for their thorough reviews and valuable comments that helped to improve many aspects of the papers published in this issue. Finally, we would like to thank the Editor-in-Chief, Ming-Yang Kao, for the opportunity to edit this special issue, and Katrina Turner and Melissa Fearon for dedicated assistance. It is our hope that the articles in this issue will be a valuable resource for researchers and will stimulate further investigations into the many areas of Algorithms and Computation research. 